\title{
Calculation of the entropy from multiparticle correlation functions
}

\author{
Brian B. Laird \\ Department of Chemistry, University of Utah, Salt Lake City, Utah 84112
}

A. D. J. Haymet

Department of Physical and Theoretical Chemistry, University of Sydney, New South Wales 2006, Australia

(Received 25 November 1991)

\begin{abstract}
We consider three approximate expressions for the entropy of a single-particle fluid that require as input only the pair-correlation function $g(r)$. By evaluating numerically these entropy estimates, for several model interparticle interactions of varying range, we examine the accuracy of these expressions compared to the "exact" entropy determined from computer simulation. With the exception of the onecomponent plasma at very low densities, the entropy of all systems studied can be approximated within $10 \%$ of the exact value for fluid densities up to $95 \%$ of the freezing density, with the agreement improving for the shorter-ranged potentials. For hard spheres, a preliminary extension to third-order terms is presented.
\end{abstract}

PACS number(s): 61.20.Gy, 65.50. $+\mathrm{m}$

\section{INTRODUCTION AND THEORY}

For a system with known pairwise-additive interactions, some thermodynamic quantities, such as the pressure and average potential energy, may be calculated exactly from knowledge of the two-particle correlation functions alone. For this reason, such quantities may be determined in a straightforward manner from computer simulations or integral equation theories. On the other hand, the corresponding expressions for important quantities such as the entropy (and, therefore, the free energy) involve not only the pair-correlation functions, but have additional contributions from multiparticle correlations of all orders. Since information about multiparticle correlations for $n \geq 3$ is either lacking or extremely difficult to obtain, it is important to know the degree to which the entropy can be approximated when the multiparticle contributions beyond the two-particle terms are ignored.

In this paper, we investigate three approximate expressions for the total entropy of a liquid. These approximations are evaluated for five model systems, the classical one-component plasma (OCP), the inverse sixth-power and inverse twelfth-power liquids, the Lennard-Jones liquid, and the hard-sphere model (both "exactly" and within the Percus-Yevick approximation). Although these approximations appear earlier in the literature $[1-3]$, we believe their importance and utility has not been understood or explained, especially in the case of the incompressible liquid approximation.

For a closed system with constant volume $V$, temperature $T$, and particle number $N$, an expression for the entropy in terms of the canonical ensemble multiparticle correlation functions was derived in 1952 by Green [4] using an expansion due to Kirkwood [5]:

$$
S_{C} / N k=s_{C}^{0}+s_{C}^{(2)}+s_{C}^{(3)}+\cdots
$$

with

$$
\begin{aligned}
& s_{C}^{0}=\frac{3}{2}-\ln \left(\rho \Lambda^{3}\right), \\
& s_{C}^{2}=-\frac{\rho}{2} \int_{V} d \mathbf{r} g_{C}^{(2)}(\mathbf{r}) \ln g_{C}^{(2)}(\mathbf{r}), \\
& s_{C}^{3}=-\frac{\rho^{2}}{6 V} \iiint_{V} d \mathbf{r}_{1} d \mathbf{r}_{2} d \mathbf{r}_{3} g_{C}^{(3)}\left(\mathbf{r}_{1}, \mathbf{r}_{2}, \mathbf{r}_{3}\right) \\
& \quad \times \ln \frac{g_{C}^{(3)}\left(\mathbf{r}_{12}, \mathbf{r}_{23}, \mathbf{r}_{13}\right)}{g_{C}^{(2)}\left(\mathbf{r}_{12}\right) g_{C}^{(2)}\left(\mathbf{r}_{23}\right) g_{C}^{(2)}\left(\mathbf{r}_{13}\right)},
\end{aligned}
$$

where $\Lambda$ in Eq. (2) is the thermal wavelength.

In 1958, Nettleton and Green [6] derived the corresponding expression for an open system (particle number not fixed), using grand-canonical correlation functions. In a much later paper, Raveché [7] presented a simpler derivation of the Nettleton-Green expression which allows for easier evaluation of the higher-order terms. To third order, this grand-canonical expression is

$$
\begin{aligned}
& \frac{S_{\mathrm{GC}}}{N k}=s_{\mathrm{GC}}^{0}+s_{\mathrm{GC}}^{(2)}+s_{\mathrm{GC}}^{(3)}+\cdots, \\
& s_{\mathrm{GC}}^{0}=\frac{s}{2}-\ln \left(\rho \Lambda^{3}\right), \\
& s_{\mathrm{GC}}^{2}=-\frac{\rho}{2} \int_{V} \operatorname{dr} g_{\mathrm{GC}}^{(2)}(\mathbf{r}) \ln g_{\mathrm{GC}}^{(2)}(\mathbf{r})+\frac{\rho}{2} \int_{V} \operatorname{dr}\left[g_{\mathrm{GC}}^{(2)}(\mathbf{r})-1\right],
\end{aligned}
$$




$$
\begin{aligned}
s_{\mathrm{GC}}^{3}= & -\frac{\rho^{2}}{6 V} \iiint_{V} \mathrm{dr}_{1} d \mathbf{r}_{2} d \mathbf{r}_{3} g_{\mathrm{GC}}^{(3)}\left(\mathbf{r}_{1}, \mathbf{r}_{2}, \mathbf{r}_{3}\right) \ln \frac{g_{\mathrm{GC}}^{(3)}\left(\mathbf{r}_{12}, \mathbf{r}_{23}, \mathbf{r}_{13}\right)}{g_{\mathrm{GC}}^{(2)}\left(\mathbf{r}_{12}\right) g_{\mathrm{GC}}^{(2)}\left(\mathbf{r}_{23}\right) t_{\mathrm{GC}}^{(2)}\left(\mathbf{r}_{13}\right)} \\
& +\frac{\rho^{2}}{6 V} \iiint_{V} d \mathbf{r}_{1} d \mathbf{r}_{2} d \mathbf{r}_{3}\left[g_{\mathrm{GC}}^{(3)}\left(\mathbf{r}_{1}, \mathbf{r}_{2}, \mathbf{r}_{3}\right)-3 g_{\mathrm{GC}}^{(2)}\left(\mathbf{r}_{1}, \mathbf{r}_{2}\right) g_{\mathrm{GC}}^{(2)}\left(\mathbf{r}_{1}, \mathbf{r}_{3}\right)+3 g_{\mathrm{GC}}^{(2)}\left(\mathbf{r}_{1}, \mathbf{r}_{2}\right)-1\right]
\end{aligned}
$$

The dependence of these expressions for the entropy of open and closed systems upon their accompanying correlation functions is not identical. The grand-canonical (open) expression contains terms that have no counterpart in the canonical (closed) expression. Superficially, this would appear to violate the principle that, in the thermodynamic limit, state quantities such as the entropy do not depend upon the statistical mechanical ensemble used. In reality, both expressions do yield identical values for the entropy; the additional terms in the expression for an open system arise from differences in the behavior of the grand-canonical and canonical correlation functions at large particle separation [8].

In the grand-canonical ensemble, the $n$-particle correlation functions $g^{(n)}\left(\mathbf{r}_{1}, \ldots, \mathbf{r}_{n}\right)$ approach unity as the distance between all of the particle pairs becomes large. On the other hand, for the canonical ensemble correlation functions, this asymptotic value differs from unity by a correction factor that is proportional to the isothermal compressibility and inversely proportional to the system size. For example, the canonical pair-correlation function exhibits the following asymptotic behavior as the pair separation is increased:

$g_{C}^{(2)}\left(\mathbf{r}_{1}, \mathbf{r}_{2}\right) \rightarrow 1-\frac{\bar{\kappa}}{N}+O\left[\frac{1}{N^{2}}\right), \quad\left|\mathbf{r}_{1}-\mathbf{r}_{2}\right| \rightarrow \infty$,

where

$$
\bar{\kappa}=-\frac{\rho}{\beta} \frac{1}{V}\left(\frac{\partial V}{\partial P}\right)_{T}
$$

is the dimensionless compressibility. Although these corrections to the canonical multiparticle correlation functions vanish as the system size goes to infinity, they contribute to the integrals over these correlation functions used to calculate the entropy [Eqs. (1)-(4)], even in the thermodynamic limit, since as $N$ increases so does the volume of integration. Such additional terms are sufficient to ensure that the entropy is the same in both ensembles.

In a recent paper, Baranyai and Evans [1] showed that the additional correction terms in the grand-canonical entropy expression, when evaluated using the canonical correlation functions, sum exactly to -1 . Thus, by adding these correction terms to the canonical entropy expression and subtracting -1 , they showed that the grand-canonical expression is, in fact, an ensemble invariant one. Such an invariant expansion simplified greatly the evaluation of the various contributions to the entropy from a computer simulation with constant number of particles, because the integrands involved are relatively short-ranged, local functions and hence it is not necessary to integrate over the entire volume of the simulation cell.
In liquid phase statistical mechanics, usually only the pair-correlation function is known for a given system. Hence the entropy expression [Eqs. (5)-(8)] is usually truncated after the second-order term, yielding the following approximate expression for the reduced excess entropy per particle:

$$
\begin{aligned}
s & \equiv \frac{S-S_{\text {ideal }}}{N k} \\
& \approx s^{(2)} \\
& =-\frac{\rho}{2} \int \mathrm{d} \mathbf{r}\left\{g^{(2)}(\mathbf{r}) \ln g^{(2)}(\mathbf{r})-\left[g^{(2)}(\mathbf{r})-1\right]\right\} .
\end{aligned}
$$

Studies of this approximation, for both the hard-sphere system by Mountain and Raveché [9] and the LennardJones system by Baranyai and Evans [1], show that it consistently overestimates the entropy except in a small region of densities near the freezing point. Later studies [10] have shown that this apparent agreement at high densities is merely the result of a fortuitous cancellation of relatively large, higher-order terms.

Hernando has shown [11] that the $g^{(n)} \ln ()$ terms in the entropy expansion vanish for $n \geq 3$ within the generalized superposition approximation (GSA) [12]. Since this approximation must be accurate for low to medium densities, the overestimation of the entropy by $s^{(2)}$ in this region must be due to the omission of the additional correction terms for $n \geq 3$.

Depending upon the density, there are at least two approximate methods for accounting for these omitted terms (at least in part). First, by splitting each higherorder correction term into a part that is identically zero within the GSA and a remainder term, Hernando [2] succeeded in summing a subset of the remainder terms to infinite order. The terms summed consist of a set of $n$ thorder ring diagrams that should give the dominant contribution at low density, since the balance of the terms are proportional to highly connected diagrams. Adding this infinite-order sum to $s^{(2)}$ yields the following "ring" approximation to the entropy:

$$
s \approx s^{(2)}+s^{\text {ring }},
$$

where

$$
\begin{array}{r}
s^{\mathrm{ring}=}=\frac{1}{(2 \rho)(2 \pi)^{3}} \int d \mathbf{k}\left\{\rho \widetilde{h}(k)-\rho^{2} \widetilde{h}^{2}(k) / 2\right. \\
-\ln [1+\rho \widetilde{h}(k)]\},
\end{array}
$$

and

$$
\widetilde{h}(\mathbf{k})=\int d \mathbf{r}[g(r)-1] e^{i \mathbf{k} \cdot \mathbf{r}} .
$$

To our knowledge, this approximation has not previously been evaluated numerically for any system. Note that 
Eq. (13) differs from the expression given in Ref. [2] due to a typographical error in the latter.

The second method for approximating the correction terms, which again requires knowledge only of the paircorrelation functions, is one that should be valid for intermediate densities high enough so that the fluid is relatively incompressible, but still low enough that the GSA is valid. In many systems, this region covers a substantial range of densities. In Fig. 1 we plot the inverse compressibility $\bar{\kappa}^{-1}$ as a function of the density (or $\gamma$ for the inverse power potentials-see Sec. III) in units of the freezing density (or freezing $\gamma$ ). As the density is increased and the liquid becomes more and more incompressible (large inverse compressibility), the density fluctuations in the grand-canonical ensemble approach zero. Therefore the grand-canonical and canonical correlation functions will become identical in this limit, as may be seen for $n=2$ in Eq. (8), where the difference between the asymptotic behavior of the "open"- and "closed"-system paircorrelation functions is proportional to the isothermal compressibility. This correspondence between two ensembles in the incompressible limit has been discussed extensively by Wallace [3], but if we are interpreting his comments correctly, their importance has not been understood fully by subsequent investigators [1]. Since the sum of all the higher-order correction terms $(n \geq 3)$ is known to be exactly $-\frac{1}{2}$ within the canonical ensemble [1], the grand-canonical entropy should approach

$$
s \approx s^{\mathrm{inc}} \equiv s^{(2)}-\frac{1}{2}
$$

in the incompressible limit, provided the density is still

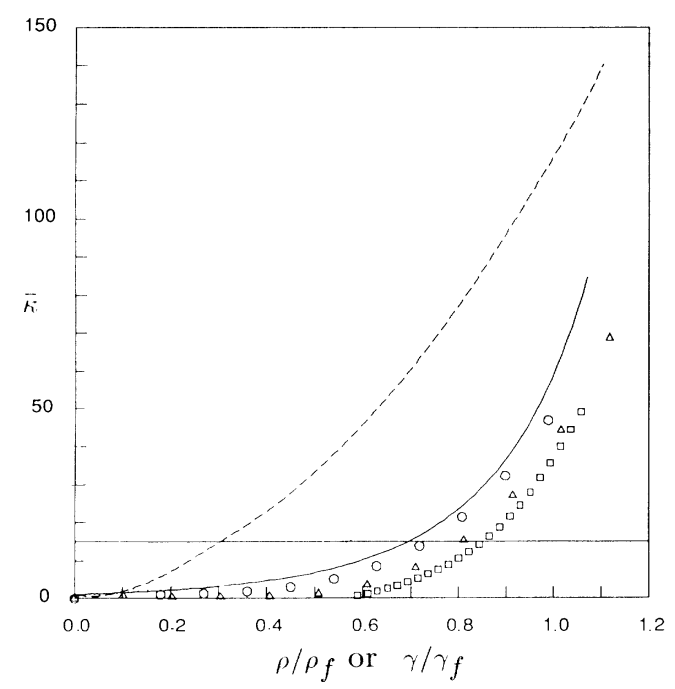

FIG. 1. Inverse (dimensionless) compressibilities of the model liquids, as a function of the density (or $\gamma$ for the inversepower potentials) relative to the value at freezing: Verlet-Weiss hard spheres (solid line), inverse 12th-power fluid (dot-dashed line), inverse sixth-power fluid (dashed line), and the $\mathrm{LJ}$ liquid at the temperatures $T^{*}=1.15$ (squares), 1.5 (triangles), and 2.74 (circles). The solid horizontal line indicates the value of the inverse compressibility at which the incompressible limit reaches the $5 \%$ accuracy level. low enough that the superposition approximation is valid. However, the question remains whether such a density region exists, and if so, how extensive it is. This we investigate below.

Note that our incompressible limit expression differs from that of Wallace [3]. We begin with the ensemble invariant $s^{(2)}$ instead of the canonical ensemble expression $s_{C}^{(2)}$ [Eq. (3)], which accounts for the $-\frac{1}{2}$ in our expression versus the -1 in Ref. [3]. Although the canonical ensemble expression does become local in the incompressible limit, it is still relatively long ranged compared to the ensemble invariant form. Hence the latter secondorder expression is more convenient for numerical evaluation [1].

In the remainder of this paper, we evaluate the above three approximations to the entropy for the following systems: hard spheres (Sec. II), inverse-power potentials (Sec. III), and the Lennard-Jones potential (Sec. IV). Comparison with the known exact entropy yields a precise calculation of the region of validity of each expression. We emphasize that in all three approximations, the only input is the two-particle correlation function. Each could be improved by addition of the appropriate threeparticle correlation function information $[13,10]$.

\section{RESULTS AND HARD SPHERES}

The hard-sphere potential, defined by

$$
v(r)= \begin{cases}0, & r / \sigma<1 \\ \infty, & r / \sigma \geq 1,\end{cases}
$$

is a natural first choice to test the various entropy expressions for several reasons. First, the interparticle potential energy for any realizable configuration is zero for this potential, and hence the excess Helmholtz free energy is purely entropic. Secondly, the existence of very good, approximate, closed-form expressions for the thermodynamic and structural properties simplifies greatly the actual numerical evaluations. In addition, due to the lack of a natural energy scale, all excess thermodynamic quantities are functions solely of the density $\rho$.

We consider two separate analytic theories for the structure and thermodynamics of hard spheres. The first is an analytic solution of the Percus-Yevick (PY) integral equation [14], derived independently by Wertheim [15] and Thiele [16]. This approximation is accurate for densities up to about $80 \%$ of the freezing density. The explicit analytic expression for the Percus-Yevick $g^{(2)}(r)$ can be found in Ref. [17]. The reduced excess entropy per particle within this approximation is

$$
s_{\mathrm{PY}}=\frac{3}{2}\left[\frac{1}{(1-\eta)^{2}}-1\right]-\ln (1-\eta),
$$

where $\eta=\pi \rho \sigma^{3} / 6$ is the packing fraction.

The second analytic expression is a fit from computersimulation data. For the thermodynamic properties, Carnahan and Starling [18] (CS) used computer-simulation results for the hard-sphere pressure to fit an analytic equation of state that is accurate even at densities near the freezing point. From this fit the excess entropy per 
particle is given by

$$
s_{\mathrm{CS}}=\frac{\eta(4-3 \eta)}{(1-\eta)^{2}}
$$

For the structure of the fluid, Verlet and Weiss [19] (VW) developed an analytic correction to the Percus-Yevick $g(r)$ that agrees well with the computer data and is thermodynamically consistent with the Carnahan-Starling equation of state.

We have calculated the simple two-body approxima-

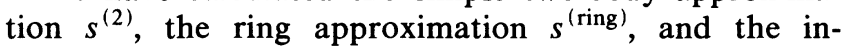
compressible approximations $s^{\text {inc }}=s^{(2)}-\frac{1}{2}$ for both these analytic expressions for the hard-sphere (HS) model. As a function of density $\rho \sigma^{3}$, these results are presented in Table I, together with the exact values calculated from Eqs. (15) and (16). To better illustrate the accuracy of each approximation, the entropy and the relative deviations of each from the exact value are plotted in Figs. 2 and 3, for the PY and CS-VW models, respectively. To facilitate comparison between the model liquids, all plots are shown in units of the density divided by the freezing density, which for hard spheres [20] is $0.943 \sigma^{3}$.

Figures 2 and 3 show that the value of the entropy given by the ring diagram expression is very good at low densities as expected. The range of this agreement is quite extensive, with a deviation of $5 \%$ or less up to half the freezing density. The pure second-order term $s^{(2)}$ consistently underestimates the entropy and gives a maximum error of about $20 \%$ at about $60 \%$ of the freezing density. Just above freezing, the error for this term decreases to zero due to the fortuitous cancellation of errors cited above.

In the incompressible limit, the approximation $s^{\text {inc }}=s^{(2)}-\frac{1}{2}$ displays the expected poor behavior at low densities, where the fluid is, in fact, compressible. A major result of this paper is the observation that as the density increases and the fluid becomes more incompressible, this deviation drastically decreases, reaching a minimum value in the region between about $70 \%$ and $90 \%$ of freezing where this approximation is nearly exact. At still

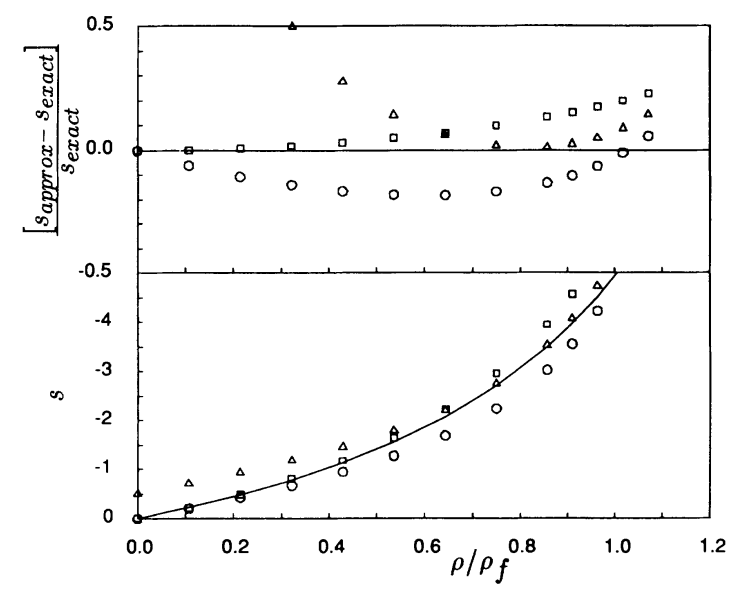

FIG. 2. The entropy (lower panel) and error relative to the exact excess entropy (upper panel) for hard spheres in the Percus-Yevick approximation, as a function of $\rho^{*} / \rho_{f}^{*}$ for three different approximations: $s^{(2)}$ (circles), $s^{(2)}+s^{\text {ring }}$ (squares), and $s$ inc (triangles). The exact excess entropy is given by the solid line in both plots. The freezing density $\rho_{f}^{*}$ for this system is $0.943[20]$.

higher densities, the relative error begins to increase again, indicating the breakdown of the superposition approximation and the growth of terms of the form $\int g^{(n)} \ln g^{(n)}$.

Recently, Baranyai and Evans (BE) [21] have calculated from Monte Carlo computer simulations of hard spheres the contribution to the ensemble invariant entropy expression due to the three-particle correlation function, $s^{(3)}$. Above a reduced density of about 0.70 , this magnitude of this three-particle term increases rapidly with density, and quickly exceeds the limiting "incompressible" value of $\frac{1}{6}$. Hence these data imply that the superposition approximation breaks down at densities lower than implied by our data in the two-body "incompressible" limit. Thus together the two sets of data imply that the accuracy of the two-body expressions at

TABLE I. Exact and estimated excess entropy for the hard-sphere fluid as a function of density. The approximations are defined in the text.

\begin{tabular}{|c|c|c|c|c|c|c|c|c|}
\hline \multirow[b]{2}{*}{$\rho^{*}$} & \multicolumn{2}{|c|}{$S_{\text {exact }}$} & \multicolumn{2}{|c|}{$s^{(2)}$} & \multicolumn{2}{|c|}{$s^{(2)}-\frac{1}{2}$} & \multicolumn{2}{|c|}{$s^{(2)}+s^{\text {ring }}$} \\
\hline & PY & VW & PY & VW & PY & VW & PY & VW \\
\hline 0.00 & 0.000 & 0.000 & 0.000 & 0.000 & -0.500 & -0.500 & 0.000 & 0.000 \\
\hline 0.10 & -0.224 & -0.224 & -0.211 & -0.211 & -0.711 & -0.711 & -0.224 & -0.224 \\
\hline 0.20 & -0.482 & -0.482 & -0.432 & -0.432 & -0.932 & -0.932 & -0.486 & -0.486 \\
\hline 0.30 & -0.782 & -0.780 & -0.673 & -0.674 & -1.173 & -1.174 & -0.796 & -0.795 \\
\hline 0.40 & -1.350 & -1.130 & -0.948 & -0.952 & -1.448 & -1.452 & -1.171 & -1.170 \\
\hline 0.50 & -1.556 & -1.544 & -1.277 & -1.284 & -1.777 & -1.784 & -1.643 & -1.629 \\
\hline 0.60 & -2.066 & -2.042 & -1.691 & -1.699 & -2.191 & -2.199 & -2.213 & -2.203 \\
\hline 0.70 & -2.694 & -2.649 & -2.243 & -2.244 & -2.743 & -2.744 & -2.959 & -2.936 \\
\hline 0.80 & -3.484 & -3.403 & -3.028 & -3.000 & -3.528 & -3.500 & -3.946 & -3.896 \\
\hline 0.85 & -3.960 & -3.851 & -3.331 & -3.498 & -3.831 & -3.998 & -4.563 & -4.491 \\
\hline 0.90 & -4.502 & -4.359 & -4.227 & -4.108 & -4.727 & -4.608 & -5.288 & -5.185 \\
\hline 0.95 & -5.126 & -4.938 & -5.082 & -4.869 & -5.582 & -5.369 & -6.148 & -6.003 \\
\hline 1.00 & -5.851 & -5.604 & -6.197 & -5.830 & -6.697 & -6.330 & -7.175 & -6.973 \\
\hline
\end{tabular}




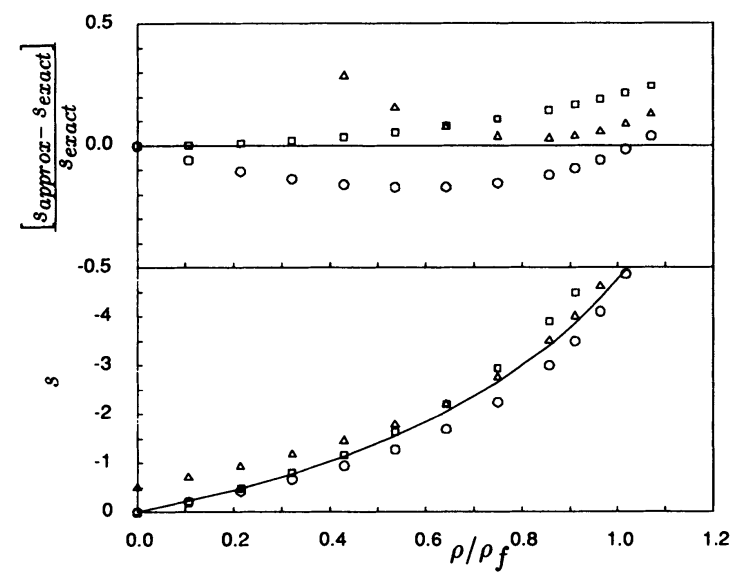

FIG. 3. Same as Fig. 2 except for the VerletWeiss-Carnahan-Starling approximation.

high densities near freezing must be the result of a fortuitous cancellation of the three-particle term by the (as yet unknown) four-body and higher multiparticle contributions.

\section{INVERSE-POWER POTENTIALS}

The inverse-power potential is defined to be

$$
v(r)=\epsilon(r / \sigma)^{n}
$$

and contains both the hard-sphere system discussed above $(n=\infty)$ and the familiar classical one-component plasma $(n=1)$ as limiting cases. This series of purely repulsive model potentials has two properties useful for the current study. First, all excess thermodynamic prop- erties can be shown [22] to be functions of a single, combined density/temperature variable

$$
\gamma n=\rho^{*}\left(T^{*}\right)^{-3 n}=\rho \sigma^{3}(k T / \epsilon)^{-3 n}
$$

Therefore the two-dimensional temperature-density space collapses onto a one-dimensional line, reducing greatly the computational work required for a thorough study.

The second important property of this potential series is the fact that, by varying $n$, the range of the potential can change dramatically. Thus we are able to study how the accuracy of each second-order entropy expression is affected by the range of the interparticle potential.

In this study, we consider three members of this potential family (in addition to the $n=\infty$ hard-sphere system examined above in Sec. III). These are the short-ranged $n=12$ "soft-sphere" fluid, the intermediate $n=6$ fluid, and the extremely long-ranged one-component plasma, $n=1$. For the $n=6$ and 12 systems, the structural and thermodynamic input for the calculation is obtained from the modified hypernetted-chain (HNC) integral equation. A description of the specific technique is given by Rosenfeld [23] and references therein. This method yields accurately both the pair-correlation function $g(r)$ and the excess Helmholtz free energy, from which the entropy can be calculated by subtracting off the average potential energy. Comparison of these results to computer simulation [24] shows that for these systems this technique is exact for all practical purposes.

The OCP system $(n=1)$ differs from the other two inverse-power systems in that a uniform neutralizing background potential is required to ensure charge neutrality and finite thermodynamic functions. For this system, the Monte Carlo computer-simulation results of

TABLE II. Exact and estimated excess entropies for the $n=12$ and 6 inverse-power systems as functions of $\gamma_{n}$.

\begin{tabular}{ccccccr}
\hline \hline$n$ & $\gamma_{n}$ & $\gamma_{n} / \gamma_{n, f}$ & \multicolumn{1}{c}{$s_{\text {exact }}$} & \multicolumn{1}{c}{$s^{(2)}$} & $s^{(2)}-\frac{1}{2}$ & $s^{(2)}+r^{\text {(ring) }}$ \\
\hline 12 & 0.00 & 0.000 & 0.00 & 0.00 & -0.50 & 0.00 \\
& 0.10 & 0.087 & -0.20 & -0.18 & -0.68 & -0.20 \\
& 0.20 & 0.174 & -0.42 & -0.36 & -0.86 & -0.43 \\
& 0.30 & 0.261 & -0.67 & -0.54 & -1.04 & -0.69 \\
& 0.40 & 0.348 & -0.94 & -0.73 & -1.23 & -0.98 \\
& 0.50 & 0.435 & -1.23 & -0.94 & -1.44 & -1.32 \\
& 0.60 & 0.522 & -1.56 & -1.19 & -1.69 & -1.71 \\
& 0.70 & 0.609 & -1.91 & -1.47 & -1.97 & -2.15 \\
& 0.80 & 0.696 & -2.28 & -1.82 & -2.32 & -2.64 \\
& 0.90 & 0.783 & -2.69 & -2.24 & -2.74 & -3.21 \\
& 1.00 & 0.870 & -3.12 & -2.75 & -3.25 & -3.84 \\
& 1.10 & 0.956 & -3.60 & -3.38 & -3.88 & -4.55 \\
& 1.20 & 1.043 & -4.09 & -4.16 & -4.66 & -5.35 \\
& & & & & \\
6 & 0.000 & 0.000 & 0.00 & 0.00 & -0.50 & -0.00 \\
& 0.316 & 0.138 & -0.58 & -0.44 & -0.94 & -1.24 \\
& 0.632 & 0.276 & -1.15 & -0.81 & -1.31 & -1.91 \\
& 0.949 & 0.414 & -1.70 & -1.20 & -1.70 & -2.61 \\
& 1.265 & 0.552 & -2.24 & -1.65 & -2.15 & -3.34 \\
1.581 & 0.690 & -2.76 & -2.17 & -2.67 & -4.09 \\
& 1.897 & 0.828 & -3.26 & -2.78 & -3.28 & -4.86 \\
& 2.214 & 0.966 & -3.76 & -3.48 & -3.98 & -5.66 \\
\hline \hline 2.530 & 1.104 & -4.26 & -4.28 & -4.78 &
\end{tabular}


TABLE III. Exact and estimated excess entropies for the one-component plasma discussed in Sec. III as a function of $\Gamma$.

\begin{tabular}{rlrrrr}
\hline \hline$\Gamma$ & $\Gamma / \Gamma_{f}$ & \multicolumn{1}{c}{$s_{\text {exact }}$} & \multicolumn{1}{c}{$s^{(2)}$} & $s^{(2)}-\frac{1}{2}$ & $s^{(2)}+s^{\text {(ring) }}$ \\
\hline 0.00 & 0.000 & 0.000 & 0.000 & -0.500 & 0.000 \\
1.00 & 0.0056 & -0.136 & -0.099 & -0.599 & -0.148 \\
5.00 & 0.0278 & -0.554 & -0.357 & -0.857 & -0.619 \\
10.00 & 0.0556 & -0.895 & -0.557 & -1.057 & -1.022 \\
15.00 & 0.0833 & -1.155 & -0.715 & -1.215 & -1.341 \\
20.00 & 0.1111 & -1.371 & -0.853 & -1.353 & -1.614 \\
30.00 & 0.1667 & -1.724 & -1.101 & -1.601 & -2.081 \\
40.00 & 0.2222 & -2.013 & -1.328 & -1.828 & -2.481 \\
60.00 & 0.3333 & -2.483 & -1.752 & -2.252 & -3.162 \\
80.00 & 0.4444 & -2.865 & -2.157 & -2.657 & -3.747 \\
100.00 & 0.5555 & -3.193 & -2.554 & -3.054 & -4.272 \\
120.00 & 0.6666 & -3.482 & -2.948 & -3.448 & -4.757 \\
130.00 & 0.7221 & -3.616 & -3.144 & -3.644 & -4.036 \\
150.00 & 0.8333 & -3.865 & -3.536 & -4.621 & \\
180.00 & 1.0000 & -4.202 & -4.121 & -4.816 & \\
190.00 & 1.0556 & -4.307 & -4.316 & & \\
\hline \hline
\end{tabular}

Stringfellow, Dewitt, and Slattery [25] are used for both the structure and thermodynamics. Following the usual convention for the OCP, all thermodynamic properties are calculated as functions of the density/temperature variable

$$
\Gamma=\frac{(Z E)^{2}}{k T}\left[\frac{4 \pi \rho}{3}\right)^{1 / 3}=\left(\frac{4 \pi \gamma_{1}}{3}\right)^{1 / 3},
$$

where $Z e$ is the charge on a given particle. Since $\Gamma$ is a function of $\gamma_{1}$, it follows from the above that all excess thermodynamic quantities are functions of $\Gamma$ alone.

For the $n=12$ and 6 potentials, the values of $s^{(2)}$, $s^{(2)}+s^{\text {ring }}$, and $s^{i n c}$ are given in Table II as functions of $\gamma_{n}$, together with the exact excess entropy from the modified HNC integral equation. For the OCP, these data are collected in Table III as a function $\Gamma$. The en-

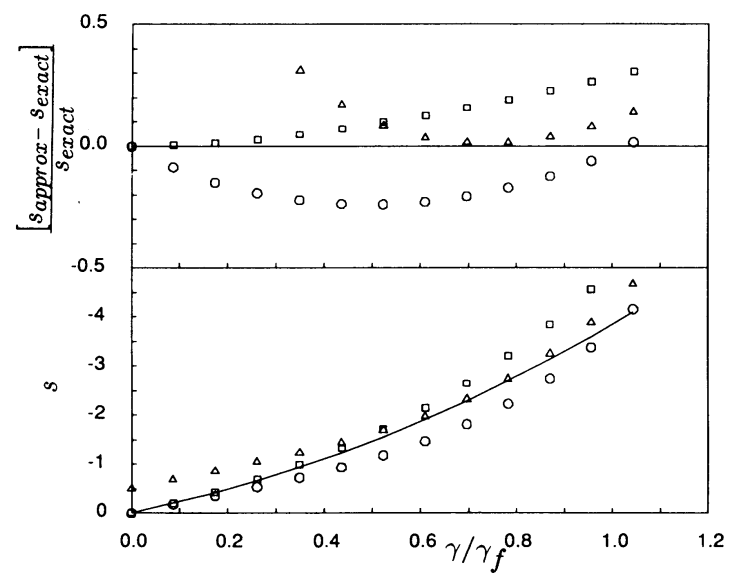

FIG. 4. The entropy (lower panel) and error relative to the exact excess entropy (upper panel) for the inverse 12th-power potential fluid as a function of $\gamma / \gamma f$ (see text for definition) for three different approximations: $s(2)$ (circles), $s^{(2)}+s^{\text {ring }}$ (squares), and $s^{\text {inc }}$ (triangles). The freezing point $\gamma_{f}$ for this system is 1.15 [20]. tropy (lower plots) and the relative error (upper plots) of each approximation are shown for the three potentials $n=12,6$, and 1 , in Figs. 4, 5, and 6, respectively. For $n=12$ and 6 , the plots are given as functions of $\gamma_{n}$ in units of the value of $\gamma_{n, f}$ at freezing, which are 1.15 [22] and 2.29 [24] for $n=12$ and 6, respectively. For the OCP system, the plots in Fig. 6 are given as functions of $\Gamma / \Gamma_{f}$, where the freezing point $\Gamma_{f}=180$ [25]. Note again that the raw data are included in the tables, so that they may be reused if more accurate freezing data become available.

Figure 4 for $n=12$ resembles closely the plots for hard spheres (Fig. 2 and 3), which is expected due to the short-ranged nature of the potential. As the range of the potential is increased, as $n$ decreases from $\infty$ (hard spheres) to 1 (OCP), three general trends emerge. First, the region of validity of the ring approximation, relative to the freezing density, is reduced. Secondly, the minimum in $s^{(2)}$ relative error (upper plots) shifts toward lower densities, with the maximum relative error increas-

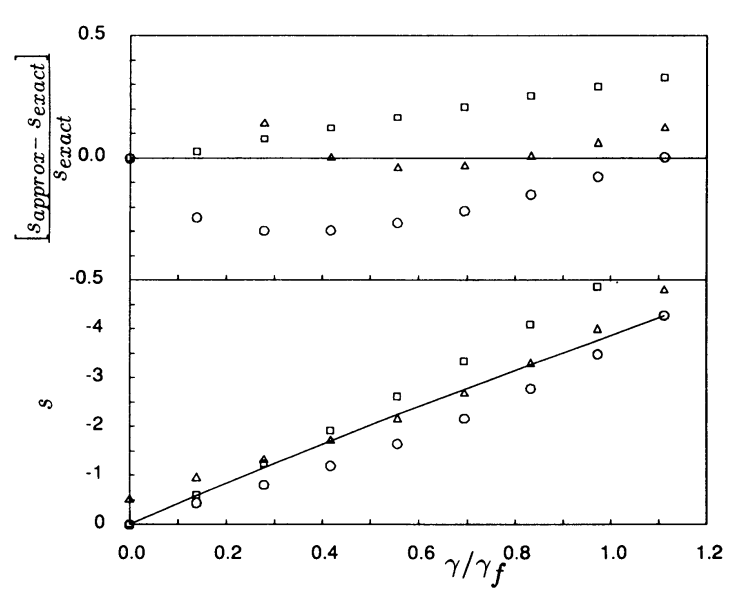

FIG. 5. Same as Fig. 4 except for the inverse sixth-power potential fluid. The freezing point $\gamma_{f}$ for this system is 2.29 [24]. 


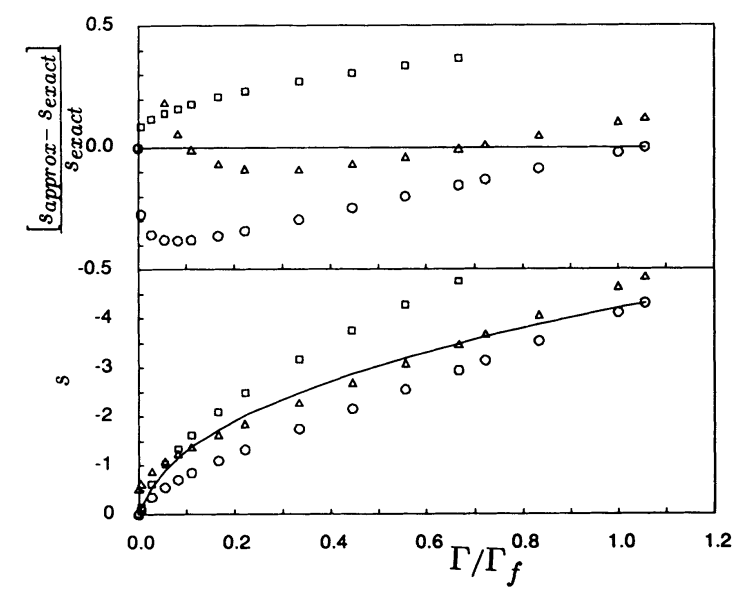

FIG. 6. The entropy (lower panel) and error relative to the exact excess entropy (upper panel) for the classical onecomponent plasma as a function $\Gamma / \Gamma_{f}$ (see text for definition) for three different approximations: $s^{(2)}$ (circles), $s^{(2)}+s^{\text {ring }}$ (squares), and $s^{\text {inc }}$ (triangles). The exact excess entropy is given by the solid line in both plots. The freezing point $\Gamma_{f}$ for this system is 180 [25].

ing from about $20 \%$ in hard spheres to almost $40 \%$ in the OCP. Finally, for the incompressible limit approximation, the location of the minimum in the relative error is shifted in the same manner as for $s^{(2)}$ but its magnitude decreases, thereby increasing the range of validity of the incompressible limit. This of course corresponds to the observed relative incompressibility of these liquids at these densities.

We note that the increase in the range of validity for $s^{\text {inc }}$ compensates for the decrease in the region of validity of the ring approximation. Hence, if one considers the function $\max \left[s^{\text {inc }}, s^{(2)}+s^{\text {ring }}\right]$ as an approximation to the entropy at all densities, one obtains a relative error of $10 \%$ or less over the full fluid and liquid range (except for the low-density OCP for which the relative error is a few percent higher).

\section{THE LENNARD-JONES LIQUID}

The final system considered is the well-known Lennard-Jones potential

$$
v(r)=4 \epsilon\left[\left(\frac{\sigma}{r}\right)^{12}-\left(\frac{\sigma}{r}\right)^{6}\right] .
$$

This potential energy is a good model for the interaction between nonpolar closed-shell atoms and molecules. The principal difference between the Lennard-Jones potential and the purely repulsive potentials considered above is the inclusion of an attractive region in the potential. This yields distinct liquid and gas phases at low temperatures, separated by a region of coexistence which becomes smaller as the temperature is raised, until it disappears at the critical temperature. Above this critical temperature, there is no longer a distinction between liquid and gas phases. As the critical point is approached, density fluc- tuations become dominant and the compressibility diverges.

For the present study, the existence of the coexistence region and the divergence of the compressibility near the critical point divides the temperature range into three regions. For temperatures between the triple point and the critical point, the dense liquid region is narrow and sandwiched between the liquid/gas and liquid/solid regions. The second regiop includes temperatures above criticality but low enough that the thermodynamic behavior is influenced by the presence of the critical point singularity. In this region, the compressibility along an isotherm is not a monotonically decreasing function of the density, but instead exhibits a maximum at the point of closest approach to the critical point. The third region covers temperatures far enough above the critical point that the isotherm is unaffected by its presence; here the compressibility is a monotonically decreasing function of density, as it is in the purely repulsive systems. Here the repulsive part of the potential is dominant and the behavior of the various approximate expressions for the entropy should be similar to that of the shorter-ranged inverse-power systems.

We study the Lennard-Jones (LJ) system at three reduced temperatures, $T^{*}=k T / \epsilon=1.15,1.50$, and 2.74 . Since the reduced critical temperature of the LennardJones system is approximately 1.32 , each temperature represents one of the three regions discussed above. The LJ structural data are obtained from an analytic fit to computer-simulation $g(r)$ data due to Goldman [26]. The exact excess entropy at $T^{*}=1.15$ and 2.74 has been evaluated by Hansen and Verlet [27] using Monte Carlo computer simulation. At $T=1.5$ thermodynamic integration using both virial coefficient data [28] and an equation of state fit by Nicholas et al. [29] is used to calculate the excess Helmholtz free energy. The excess entropy is then calculated by subtracting this free energy from the average potential energy, determined from the

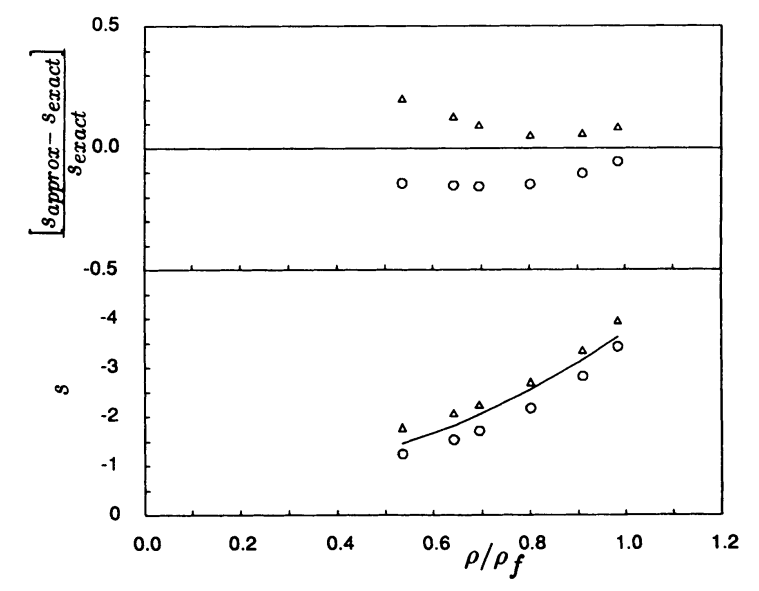

FIG. 7. The entropy (lower panel) and error relative to the exact excess entropy (upper panel) for the Lennard-Jones liquid at $T^{*}=1.15$ as a function of $\rho^{*} / \rho_{f}^{*}$ for two different approximations: $s^{(2)}$ (circles) and $s^{\text {inc }}$ (triangles). The exact excess entropy is given by the solid line in both plots. The freezing density $\rho_{f}^{*}$ for this system is 0.936 [27]. 


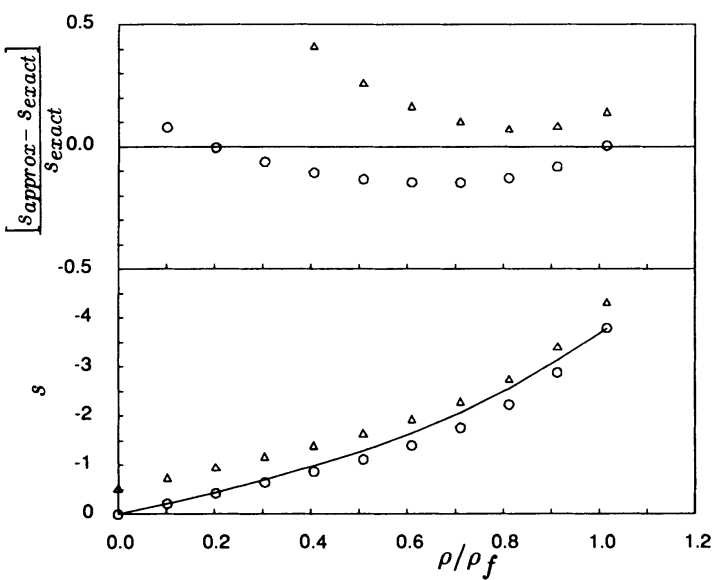

FIG. 8. Same as Fig. 7 except for $T^{*}=1.50$ The freezing density $\rho_{f}^{*}$ for this system is $0.985[27]$.

\section{Goldman structural data.}

The calculated values of $s^{(2)}$ and $s^{\text {inc }}$ together with the exact excess entropy for all three temperatures are listed in Table IV as a function of reduced density, $\rho^{*}=\rho \sigma^{3}$. The ring approximation is not calculated, since the Fourier transform of $g(r)$ is not known to sufficient accuracy to yield meaningful results. The excess entropy (lower plots) and relative error (upper plots) from these two approximations are plotted in Figs. 7, 8, and 9 for

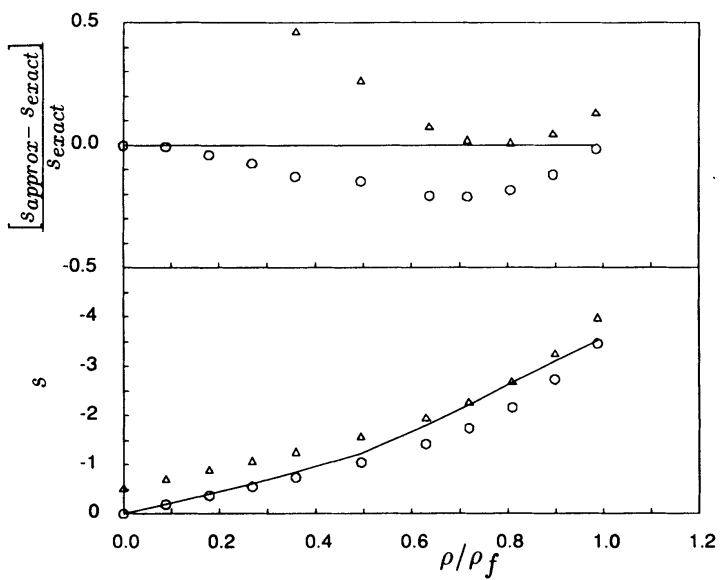

FIG. 9. Same as Fig. 7 except for $T^{*}=2.74$ The freezing density $\rho_{f}^{*}$ for this system is 1.113 [27].

$T^{*}=1.15,1.5$, and 2.74 , respectively, as a function of the reduced density in units of the reduced freezing density. From computer simulation, the reduced freezing densities for $T^{*}=1.15,1.5$, and 2.74 are $0.936,0.985$, and 1.113 , respectively [27] (where the value for 1.50 has been interpolated).

For $T^{*}=2.74$, Fig. 9 is very similar to the data for hard spheres and the inverse 12th-power potential, as expected from the similarity of the potentials. For the

TABLE IV. Exact and estimated excess entropy for the Lennard-Jones system as a function of density for a variety of temperatures.

\begin{tabular}{|c|c|c|c|c|c|}
\hline$T^{*}$ & $\rho$ & $\rho / \rho_{f}$ & $s_{\mathrm{MC}}$ & $s^{(2)}$ & $s^{(2)}-\frac{1}{2}$ \\
\hline \multirow[t]{6}{*}{1.15} & 0.55 & 0.558 & -1.457 & -1.25 & -1.75 \\
\hline & 0.60 & 0.642 & -1.815 & -1.54 & -2.04 \\
\hline & 0.65 & 0.695 & -2.037 & -1.72 & -2.22 \\
\hline & 0.75 & 0.802 & -2.556 & -2.18 & -2.68 \\
\hline & 0.85 & 0.910 & -3.150 & -2.83 & -3.33 \\
\hline & 0.92 & 0.984 & -3.625 & -3.43 & -3.93 \\
\hline \multirow[t]{11}{*}{1.50} & 0.00 & 0.000 & 0.000 & 0.000 & -0.500 \\
\hline & 0.10 & 0.102 & -0.480 & -0.216 & -0.716 \\
\hline & 0.20 & 0.203 & -0.917 & -0.430 & -0.930 \\
\hline & 0.30 & 0.304 & -1.290 & -0.648 & -1.148 \\
\hline & 0.40 & 0.406 & -1.657 & -0.873 & -1.373 \\
\hline & 0.50 & 0.508 & -2.023 & -1.119 & -1.619 \\
\hline & 0.60 & 0.609 & -2.417 & -1.405 & -1.905 \\
\hline & 0.70 & 0.711 & -2.848 & -1.761 & -2.261 \\
\hline & 0.80 & 0.812 & -3.334 & -2.231 & -2.731 \\
\hline & 0.90 & 0.913 & -3.840 & -2.885 & -3.385 \\
\hline & 1.00 & 1.020 & -4.500 & -3.794 & -4.294 \\
\hline \multirow[t]{11}{*}{2.74} & 0.00 & 0.000 & 0.000 & 0.000 & -0.500 \\
\hline & 0.10 & 0.089 & -0.193 & -0.192 & -0.692 \\
\hline & 0.20 & 0.179 & -0.392 & -0.375 & -0.875 \\
\hline & 0.30 & 0.269 & -0.600 & -0.556 & -1.056 \\
\hline & 0.40 & 0.358 & -0.855 & -0.743 & -1.243 \\
\hline & 0.55 & 0.494 & -1.232 & -1.052 & -1.552 \\
\hline & 0.70 & 0.637 & -1.801 & -1.431 & -1.931 \\
\hline & 0.80 & 0.716 & -2.215 & -1.752 & -2.252 \\
\hline & 0.90 & 0.806 & -2.654 & -2.169 & -2.669 \\
\hline & 1.00 & 0.895 & -3.108 & -2.735 & -3.235 \\
\hline & 1.10 & 0.985 & -3.511 & -3.460 & -3.960 \\
\hline
\end{tabular}


lower two temperatures, the $s^{\text {inc }}$ error minima (upper plots) are shifted slightly to higher densities and do not intersect or touch the exact curve as do the other plots. We interpret this as confirmation that the superposition approximation breaks down at lower (reduced) densities [30].

\section{DISCUSSION}

Several general trends are observed in the data. First, the range of applicability of the "ring" expression decreases as the range of interparticle interaction increases. The origin of this trend is due probably to the highly connected diagrams that are omitted from this approximation. These terms, like the "ring" diagrams, are nonzero within the GSA and will contribute at moderate densities. As the interaction range increases, the relative importance of such terms will increase, and hence decrease the accuracy of the ring expression at a fixed density.

Secondly, the error in the incompressible limit approximation, $s$ inc , has the same general behavior for all systems. At low densities, the deviation from the exact excess entropy is large, due to the large compressibility of the system. As the density is increased, this deviation decreases until it reaches a minimum and begins to increase again. For the systems studied, when the value of the dimensionless inverse compressibility, $\bar{\kappa}^{-1}$, reaches about 15 , the accuracy of the incompressible limit expression for the excess entropy is approximately $5 \%$ (see Fig. 1). For the short-ranged systems, the magnitude of the deviation is nearly zero at the minimum, and the approximation is nearly exact for a large region of densities just below freezing. At densities near freezing, the breakdown of the superposition approximation causes the deviation to rise. As the range of the interaction is increased, the minimum in the relative error is shifted toward lower densities relative to the freezing density. Moreover it is displaced downward so that it intersects the exact curve instead of being tangent to it as with the shorter-ranged potentials. The shifting of the minimum to lower densities is due to the fact that the compressibility at a given density relative to the freezing density decreases as the range of the interaction is increased, thereby lowering the densities at which this approximation begins to be valid, thus compensating for the decrease in the range of validity of the ring approximation.

In summary, from the above comparisons for various systems in the previous three sections, we see that the ring approximation together with the incompressible limit expression yields substantial improvement over the simple second-order truncation expression $s^{(2)}$ in the calculation of the excess entropy, while still requiring only the very same input, namely, the pair-correlation function. By constructing an excess entropy function that is equal to the larger (that is, less negative) of $s^{(2)}+s^{\text {ring }}$ and $s$ inc, the excess entropy of the fluid for all systems studied (except the OCP at very low densities) is estimated to within $10 \%$ or less for densities below about $95 \%$ of the freezing density.

As discussed in Sec. II, recent calculations of the three-body contribution to the entropy, $s^{(3)}$, for hard spheres by Baranyai and Evans [21] shows that the relative accuracy of the two-particle entropy expression at densities near the freezing point is significantly diminished upon addition of the three-particle term. Therefore the success of the two-particle results at these densities seems to be due to a fortuitous and, judging from our present results, seemingly universal cancellation of the three-body terms by the four-body and higher multiparticle contributions. Further analysis of the higher-order terms and the nature of this cancellation of errors is thus warranted and is underway.

\section{ACKNOWLEDGMENTS}

This research was supported by the U.S. National Science Foundation through Grant No. CHE-8913006. A grant of computer time from the Utah Supercomputing Institute (USI), funded by Utah and IBM Corporation, is acknowledged gratefully. We also thank Dr. Douglas Henderson for the use of his computer program to evaluate the hard-sphere pair-correlation function
[1] A. Baranyai and D. J. Evans, Phys. Rev. A 40, 3817 (1989).

[2] J. A. Hernando, Mol. Phys. 69, 327 (1990).

[3] D. C. Wallace, J. Chem. Phys. 87, 2282 (1987); Phys. Rev. A 39, 4843 (1989).

[4] H. S. Green, The Molecular Theory of Fluids (NorthHolland, Amsterdam, 1952).

[5] J. G. Kirkwood, J. Chem. Phys. 10, 394 (1942).

[6] R. E. Nettleton and H. S. Green, J. Chem. Phys. 29, 1365 (1958).

[7] H. J. Raveché, J. Chem. Phys. 55, 2242 (1971).

[8] J. L. Lebowitz and J. K. Percus, Phys. Rev. 122, 1675 (1961).

[9] R. D. Mountain and H. J. Raveché, J. Chem. Phys. 55, 2250 (1971).

[10] A. Baranyai and D. J. Evans, Phys. Rev. A 42, 849 (1990).

[11] J. A. Hernando, Mol. Phys. 69, 319 (1990).

[12] I. Z. Fisher and B. L. Kopeliovich, Dokl. Akad. Nauk
SSSR 133, 81 (1961) [Sov. Phys. - Dokl. 5, 761 (1961)].

[13] A. D. J. Haymet, J. Phys. (Paris) Colloq. 46, C9-27 (1985).

[14] J. K. Percus and G. J. Yevick, Phys. Rev. 110, 1 (1958).

[15] M. S. Wertheim, Phys. Rev. Lett. 10, 321 (1963).

[16] E. Thiele, J. Chem. Phys. 39, 474 (1963).

[17] D. Henderson and E. W. Grundke, J. Chem. Phys. 63, 601 (1975).

[18] N. F. Carnahan and K. E. Starling, J. Chem. Phys. 51, 635 (1969).

[19] L. Verlet and J. Weiss, Phys. Rev. A 5, 939 (1972).

[20] W. G. Hoover and F. M. Ree, J. Chem. Phys. 49, 3609 (1968).

[21] A. Baranyai and D. J. Evans, Z. Naturforsch. Teil A 46, 27 (1990).

[22] W. G. Hoover, M. Ross, K. W. Johnson, D. Henderson, J. A. Barker, and B. C. Brown, J. Chem. Phys. 52, 4931 (1970).

[23] Y. Rosenfeld, J. Stat. Phys. 42, 437 (1986). 
[24] B. B. Laird and A. D. J. Haymet, Mol. Phys. 75, 71 (1992).

[25] G. W. Stringfellow, H. E. DeWitt, and W. L. Slattery, Phys. Rev. A 41, 1105 (1990).

[26] S. Goldman, J. Phys. Chem. 83, 3033 (1979).

[27] J. P. Hansen and L. Verlet, Phys. Rev. 184, 151 (1969).

[28] J. A. Barker, P. J. Leonard, and A. Pompe, J. Chem. Phys.
44, 4206 (1966).

[29] J. J. Nicholas, K. E. Gubbins, W. B. Streett, and D. J. Tildesley, Mol Phys. 37, 1429 (1979).

[30] W. J. McNeil, W. G. Madden, A. D. J. Haymet, and S. A. Rice, J. Chem. Phys. 78, 388 (1983). 